\title{
Dirichlet problem for divergence form elliptic equations with discontinuous coefficients
}

\author{
Sara Monsurrò and Maria Transirico*
}

"Correspondence:

mtransirico@unisa.it

Dipartimento di Matematica,

Università di Salerno, via Ponte Don

Melillo, Fisciano (SA), 84084, Italy

\begin{abstract}
We study the Dirichlet problem for linear elliptic second order partial differential equations with discontinuous coefficients in divergence form in unbounded domains. We establish an existence and uniqueness result and we prove an a priori bound in $L^{p}, p>2$.
\end{abstract}

MSC: $35 \mathrm{~J} 25 ; 35 \mathrm{~B} 45 ; 35 \mathrm{R} 05$

Keywords: elliptic equations; discontinuous coefficients; a priori bounds

\section{Introduction}

We are interested in the Dirichlet problem

$$
\left\{\begin{array}{l}
u \in \mathscr{O}^{1,2}(\Omega) \\
L u=f, \quad f \in W^{-1,2}(\Omega)
\end{array}\right.
$$

where $\Omega$ is an unbounded open subset of $\mathbb{R}^{n}, n \geq 2$, and $L$ is a linear uniformly elliptic second order differential operator with discontinuous coefficients in divergence form

$$
L=-\sum_{i, j=1}^{n} \frac{\partial}{\partial x_{j}}\left(a_{i j} \frac{\partial}{\partial x_{i}}+d_{j}\right)+\sum_{i=1}^{n} b_{i} \frac{\partial}{\partial x_{i}}+c .
$$

If $\Omega$ is bounded, this problem is classical in literature and has been deeply analyzed taking into account various kinds of hypotheses on the coefficients (for more details see, for instance, [1-6]).

Considering unbounded domains, as far as we know, the first work on this subject goes back to [7], where Bottaro and Marina provide, for $n \geq 3$, an existence and uniqueness result for the solution of problem (1.1) assuming that

$$
\begin{aligned}
& a_{i j} \in L^{\infty}(\Omega), \quad i, j=1, \ldots, n, \\
& b_{i}, d_{i} \in L^{n}(\Omega), \quad i=1, \ldots, n, \quad c \in L^{n / 2}(\Omega)+L^{\infty}(\Omega), \\
& c-\sum_{i=1}^{n}\left(d_{i}\right)_{x_{i}} \geq \mu, \quad \mu \in \mathbb{R}_{+} .
\end{aligned}
$$

In this order of ideas, various generalizations have been performed still maintaining hypotheses (1.3) and (1.5) but weakening the condition (1.4). Indeed in [8], where the case

\section{囪 Springer}

c) 2012 Monsurrò and Transirico; licensee Springer. This is an Open Access article distributed under the terms of the Creative Commons Attribution License (http://creativecommons.org/licenses/by/2.0), which permits unrestricted use, distribution, and reproduction in any medium, provided the original work is properly cited. 
$n \geq 2$ is considered, $b_{i}, d_{i}$ and $c$ are supposed to satisfy assumptions as those in (1.4), but just locally. Successively in [9], for $n \geq 3$, further improvements have been carried on since $b_{i}, d_{i}$ and $c$ are in suitable Morrey-type spaces with lower summabilities.

In [7-9] we also find the bound

$$
\|u\|_{W^{1,2}(\Omega)} \leq C\|f\|_{W^{-1,2}(\Omega)},
$$

where the dependence of the constant $C$ on the data of the problem is fully determined.

More recently, in [10], supposing that the coefficients of lower-order terms are as in [9] for $n \geq 3$ and as in [8] for $n=2$, we showed that, for a sufficiently regular set $\Omega$, and if $f \in L^{2}(\Omega) \cap L^{\infty}(\Omega)$, then there exists a constant $C$, whose dependence is completely described, such that

$$
\|u\|_{L^{p}(\Omega)} \leq C\|f\|_{L^{p}(\Omega)},
$$

for any bounded solution $u$ of (1.1) and for every $p \in] 2,+\infty[$.

Here, in the same framework but replacing the classical hypothesis of sign (1.5) by the less common one

$$
c-\sum_{i=1}^{n}\left(b_{i}\right)_{x_{i}} \geq \mu, \quad \mu \in \mathbb{R}_{+}
$$

we establish two kinds of results for the solution of (1.1). First of all, we provide an existence and uniqueness theorem, then, taking into account an additional assumption on the regularity of the boundary of $\Omega$, we prove the analogue of (1.7).

Let us briefly survey the way these results are achieved. In Section 2, we introduce the tools needed in the sequel. The definitions and some features of the Morrey-type spaces are given and some functions $u_{s}$, related somehow to the solution of the problem and to the coefficients of the operator, are described, together with some specific properties. Section 3 is devoted to the solvability of problem (1.1). We start proving, by means of the above mentioned functions $u_{s}$, the estimate in (1.6) that leads also to the uniqueness at once. Then, in view of well-known results of the operator theory, we get the existence verifying that $L$ is a Fredholm operator with zero index. In the last section, we prove the claimed $L^{p}$-estimate. This is done by means of a technical lemma, exploiting again the functions $u_{s}$, which allows us to conclude.

Considering the case $p=2$, we notice that, as a consequence of (1.6), the bound (1.7) is true under both sign hypotheses even supposing no regularity on the boundary of $\Omega$.

We believe that the two estimates (1.7), obtained under the different sign assumptions, combined together should permit to prove, by means of a duality argument, that (1.7) holds true actually for any $p \in] 1,+\infty[$, considering one of the hypotheses (1.5) or (1.8) at a time.

For further studies of the Dirichlet problem for linear elliptic second order differential equations with discontinuous coefficients in divergence form in unbounded domains we refer the reader also to [11-13]. 


\section{Tools}

This section is devoted to the definitions and to some fundamental properties of the Morrey-type spaces where the coefficients of lower-order terms of our operator belong, and of some functions $u_{s}$ related to the solution of the problem and to all the coefficients of the operator (see the proofs of Theorem 3.1 and Lemma 4.1 for more details on this aspect) that are indispensable tools in the sequel.

Given an unbounded open subset $\Omega$ of $\mathbb{R}^{n}, n \geq 2$, we denote by $\Sigma(\Omega)$ the $\sigma$-algebra of all Lebesgue measurable subsets of $\Omega$. For any $E \in \Sigma(\Omega), \chi_{E}$ is its characteristic function and $E(x, r)$ is the intersection $E \cap B(x, r)\left(x \in \mathbb{R}^{n}, r \in \mathbb{R}_{+}\right)$, where $B(x, r)$ is the open ball centered in $x$ and with radius $r$.

For $q \in\left[1,+\infty\left[\right.\right.$ and $\lambda \in\left[0, n\left[\right.\right.$, the space of Morrey type $M^{q, \lambda}(\Omega)$ is the set of all the functions $g$ in $L_{l o c}^{q}(\bar{\Omega})$ such that

$$
\|g\|_{M^{q, \lambda}(\Omega)}=\sup _{\substack{\tau \in] 0,1] \\ x \in \Omega}} \tau^{-\lambda / q}\|g\|_{L^{q}(\Omega(x, \tau))}<+\infty
$$

endowed with the norm above defined. Moreover, $M_{\circ}^{q, \lambda}(\Omega)$ denotes the closure of $C_{\circ}^{\infty}(\Omega)$ in $M^{q, \lambda}(\Omega)$. These functional spaces generalize the classical notion of Morrey spaces to the case of unbounded domains and were introduced in [9] (we refer also to [14] where further characteristics are considered).

For the reader's convenience, in the next lemma we recall some results of [15] and $[8,9]$ concerning the multiplication operator

$$
u \in \stackrel{\circ}{W}^{1,2}(\Omega) \rightarrow g u \in L^{2}(\Omega)
$$

where the function $g$ belongs to suitable spaces of Morrey type.

Lemma 2.1 If $g \in M^{q, \lambda}(\Omega)$, with $q>2$ and $\lambda=0$ if $n=2$, and $\left.\left.q \in\right] 2, n\right]$ and $\lambda=n-q$ if $n>2$, then the operator in (2.1) is bounded and there exists a constant $c \in \mathbb{R}_{+}$such that

$$
\|g u\|_{L^{2}(\Omega)} \leq c\|g\|_{M^{q, \lambda}(\Omega)}\|u\|_{W^{1,2}(\Omega)} \quad \forall u \in \stackrel{\circ}{W}^{1,2}(\Omega),
$$

with $c=c(n, q)$.

Moreover, if $g \in M_{\circ}^{q, \lambda}(\Omega)$, then the operator in (2.1) is also compact.

Now, let us deal with the above mentioned functions $u_{s}$. They were employed for the first time in [7] and were studied in the framework of Morrey-type spaces in [9].

For $h>k \geq 0$, we define the functions of the real variable $t$

$$
G_{k \infty}(t)= \begin{cases}t-k & \text { if } t>k, \\ 0 & \text { if }-k \leq t \leq k, \\ t+k & \text { if } t<-k,\end{cases}
$$

and

$$
G_{k h}(t)=G_{k \infty}(t)-G_{h \infty}(t) .
$$


Lemma 2.2 Let $g \in M_{o}^{q, \lambda}(\Omega), u \in \stackrel{\circ}{W}^{1,2}(\Omega)$ and $\varepsilon \in \mathbb{R}_{+}$. Then there exist $r \in \mathbb{N}$ and $k_{1}, \ldots, k_{r} \in \mathbb{R}$, with $0=k_{r}<k_{r-1}<\cdots<k_{1}<k_{0}=+\infty$, such that set

$$
u_{s}=G_{k_{s} k_{s-1}}(u), \quad s=1, \ldots, r
$$

one has $u_{1}, \ldots, u_{r} \in \stackrel{\circ}{W}^{1,2}(\Omega)$ and

$$
\begin{aligned}
& u=u_{1}+\cdots+u_{r}, \\
& u_{s}^{2} \leq u u_{s}, \quad s=1, \ldots, r, \\
& \left|u_{s}\right| \leq|u|, \quad s=1, \ldots, r, \\
& u_{x_{i}}\left(u_{s}\right)_{x_{j}}=\left(u_{s}\right)_{x_{i}}\left(u_{s}\right)_{x_{j}}, \quad s=1, \ldots, r, i, j=1, \ldots, n, \\
& u\left(u_{s}\right)_{x_{i}}=\left(u_{s}+\cdots+u_{r}\right)\left(u_{s}\right)_{x_{i}}, \quad s=1, \ldots, r, i=1, \ldots, n, \\
& \left\|g \chi_{\operatorname{supp}\left(u_{s}\right)_{x}}\right\|_{M^{q, \lambda}(\Omega)} \leq \varepsilon, \quad s=1, \ldots, r, \\
& r \leq c,
\end{aligned}
$$

with $c=c\left(\varepsilon, q,\|g\|_{M^{q, \lambda}(\Omega)}\right)$ positive constant.

Proof The proofs of the properties (2.6), (2.7), (2.9), (2.11) and (2.12) can be found in [9]. Inequality (2.8) is an immediate consequence of (2.7).

Considering (2.10), observe that in the case $s=1$ it is a trivial consequence of (2.6).

Thus let us fix $s \in \mathbb{N}$ and such that $2 \leq s \leq r$. As already proved in [16] and in [7], in the case of unbounded domains, one has

$$
\left(G_{k_{s} k_{s-1}}(u)\right)_{x_{i}}=G_{k_{s} k_{s-1}}^{\prime}(u) u_{x_{i}}, \quad \text { a.e. in } \Omega, i=1, \ldots, n \text {. }
$$

This, together with (2.3) and (2.4), gives

$$
\operatorname{supp}\left(u_{s}\right)_{x_{i}} \subseteq \overline{\left\{x \in \Omega \text { s.t. } k_{s}<|u|<k_{s-1}, u_{x_{i}} \neq 0\right\}},
$$

$i=1, \ldots, n$.

On the other hand, by definition,

$$
\operatorname{supp} u_{h} \subseteq\left\{x \in \Omega \text { s.t. }|u| \geq k_{h}\right\}, \quad h=1, \ldots, r .
$$

Combining (2.14) and (2.13), we conclude that

$$
\operatorname{supp} u_{h} \cap \operatorname{supp}\left(u_{s}\right)_{x_{i}}=\emptyset,
$$

$h=1, \ldots, s-1, i=1, \ldots, n$. Hence by $(2.6)$ we get $(2.10)$.

\section{Existence and uniqueness result}

Let $\Omega$ be an unbounded open subset of $\mathbb{R}^{n}, n \geq 2$. 
We are interested in the study of the following Dirichlet problem in $\Omega$ :

$$
\left\{\begin{array}{l}
u \in \stackrel{\circ}{W}^{1,2}(\Omega) \\
L u=f, \quad f \in W^{-1,2}(\Omega)
\end{array}\right.
$$

where $L$ is a second order linear differential operator in divergence form

$$
L=-\sum_{i, j=1}^{n} \frac{\partial}{\partial x_{j}}\left(a_{i j} \frac{\partial}{\partial x_{i}}+d_{j}\right)+\sum_{i=1}^{n} b_{i} \frac{\partial}{\partial x_{i}}+c,
$$

satisfying the following hypotheses on the leading coefficients:

$$
\left\{\begin{array}{l}
a_{i j} \in L^{\infty}(\Omega), \quad i, j=1, \ldots, n, \\
\exists v>0: \quad \sum_{i, j=1}^{n} a_{i j} \xi_{i} \xi_{j} \geq v|\xi|^{2} \quad \text { a.e. in } \Omega, \forall \xi \in \mathbb{R}^{n} .
\end{array}\right.
$$

Considering the coefficients of lower-order terms, we suppose that

$$
\begin{aligned}
& \left\{\begin{array}{l}
b_{i}, d_{i} \in M^{2 t, \lambda}(\Omega), \quad d_{i}-b_{i} \in M_{o}^{2 t, \lambda}(\Omega), \quad i=1, \ldots, n, \\
c \in M^{t, \lambda}(\Omega), \\
\text { with } t>1 \text { and } \lambda=0 \text { if } n=2, \\
\text { with } t \in] 1, n / 2] \text { and } \lambda=n-2 t \text { if } n>2,
\end{array}\right. \\
& \left\{\begin{array}{l}
c-\sum_{i=1}^{n}\left(b_{i}\right)_{x_{i}} \geq \mu, \quad \mu=\text { constant }>0, \\
\text { in the sense of distributions on } \Omega .
\end{array}\right.
\end{aligned}
$$

We associate to $L$ the bilinear form

$$
a(u, v)=\int_{\Omega}\left(\sum_{i, j=1}^{n}\left(a_{i j} u_{x_{i}}+d_{j} u\right) v_{x_{j}}+\left(\sum_{i=1}^{n} b_{i} u_{x_{i}}+c u\right) v\right) d x,
$$

$u, v \in \stackrel{\circ}{W}^{1,2}(\Omega)$.

As a consequence of Lemma 2.1, $a$ is continuous on $\stackrel{\circ}{W}^{1,2}(\Omega) \times \stackrel{\circ}{W}^{1,2}(\Omega)$; and therefore, the operator $L: W^{1,2}(\Omega) \rightarrow W^{-1,2}(\Omega)$ is continuous too.

Theorem 3.1 Under hypotheses $\left(h_{1}\right)-\left(h_{3}\right)$, problem (3.1) is uniquely solvable and its solution u satisfies the estimate

$$
\|u\|_{W^{1,2}(\Omega)} \leq C\|f\|_{W^{-1,2}(\Omega)},
$$

where $C$ is a constant depending on $n, t, v, \mu,\left\|d_{i}-b_{i}\right\|_{M^{2 t, \lambda}(\Omega)}, i=1, \ldots, n$.

Proof We start proving estimate (3.4) that yields also to the uniqueness of the solution at once. Successively, in view of classical results concerning operator theory, to get the existence, it will be enough to verify that $L$ is a Fredholm operator with zero index. 
Let $u_{s}$, for $s=1, \ldots, r$, be the functions of Lemma 2.2 corresponding to a solution $u$ of (3.1), to $g=\sum_{i=1}^{n}\left|d_{i}-b_{i}\right|$ and to a positive real number $\varepsilon$ that will be specified in the sequel. By a well-known characterization of the space $W^{-1,2}(\Omega)$, we have

$$
f=f_{0}-\sum_{i=1}^{n}\left(f_{i}\right)_{x_{i}}, \quad f_{k} \in L^{2}(\Omega), k=0, \ldots, n .
$$

Thus, if we take $u_{s}$ as a test function in the variational formulation of problem (3.1), by simple calculations and (2.9) and (2.10), we obtain

$$
\begin{aligned}
& \int_{\Omega} f_{0} u_{s} d x+\sum_{i=1}^{n} \int_{\Omega} f_{i}\left(u_{s}\right)_{x_{i}} d x \\
& =a\left(u, u_{s}\right) \\
& =\int_{\Omega}\left[\sum_{i, j=1}^{n} a_{i j} u_{x_{i}}\left(u_{s}\right)_{x_{j}}+\sum_{i=1}^{n}\left(d_{i} u\left(u_{s}\right)_{x_{i}}+b_{i} u_{x_{i}} u_{s}\right)+c u u_{s}\right] d x \\
& =\int_{\Omega}\left[\sum_{i, j=1}^{n} a_{i j} u_{x_{i}}\left(u_{s}\right)_{x_{j}}+\sum_{i=1}^{n} b_{i}\left(u u_{s}\right)_{x_{i}}+c u u_{s}+\sum_{i=1}^{n}\left(d_{i}-b_{i}\right) u\left(u_{s}\right)_{x_{i}}\right] d x \\
& =\int_{\Omega}\left[\sum_{i, j=1}^{n} a_{i j}\left(u_{s}\right)_{x_{i}}\left(u_{s}\right)_{x_{j}}+\sum_{i=1}^{n} b_{i}\left(u u_{s}\right)_{x_{i}}+c u u_{s}\right. \\
& \left.\quad+\sum_{i=1}^{n}\left(d_{i}-b_{i}\right)\left(\sum_{h=s}^{r} u_{h}\right)\left(u_{s}\right)_{x_{i}}\right] d x .
\end{aligned}
$$

Hypotheses $\left(h_{1}\right)$ and $\left(h_{3}\right)$ together with $(2.7)$ give then

$$
\begin{aligned}
\int_{\Omega} f_{0} u_{s} d x+\sum_{i=1}^{n} \int_{\Omega} f_{i}\left(u_{s}\right)_{x_{i}} d x \geq & v \int_{\Omega}\left(u_{s}\right)_{x}^{2} d x+\mu \int_{\Omega}\left(u_{s}\right)^{2} d x \\
& -\int_{\Omega} \sum_{h=s}^{r}\left|u_{h}\right| \sum_{i=1}^{n}\left|d_{i}-b_{i}\right|\left|\left(u_{s}\right)_{x_{i}}\right| d x .
\end{aligned}
$$

On the other hand, by the Hölder inequality, the embedding results contained in Lemma 2.1 and using hypothesis $\left(h_{2}\right)$ and (2.11), one has that there exists a constant $c_{0} \in \mathbb{R}_{+}$ such that

$$
\begin{aligned}
& \int_{\Omega} \sum_{h=s}^{r}\left|u_{h}\right| \sum_{i=1}^{n}\left|d_{i}-b_{i}\right|\left|\left(u_{s}\right)_{x_{i}}\right| d x \\
& \leq \sum_{h=s}^{r}\left\|\left|u_{h}\right| g \chi_{\operatorname{supp}\left(u_{s}\right)_{x}}\right\|_{L^{2}(\Omega)}\left\|\left(u_{s}\right)_{x}\right\|_{L^{2}(\Omega)} \\
& \leq c_{0} \sum_{h=s}^{r}\left\|u_{h}\right\|_{W^{1,2}(\Omega)}\left\|g \chi_{\operatorname{supp}\left(u_{s}\right)_{x}}\right\|_{M^{2 t, \lambda}(\Omega)}\left\|u_{s}\right\|_{W^{1,2}(\Omega)} \\
& \quad \leq \varepsilon c_{0}\left\|u_{s}\right\|_{W^{1,2}(\Omega)} \sum_{h=s}^{r}\left\|u_{h}\right\|_{W^{1,2}(\Omega)},
\end{aligned}
$$


with $c_{0}=c_{0}(n, t)$.

Hence, set

$$
\mu_{0}=\min \{v, \mu\},
$$

by (3.5) we get

$$
\begin{aligned}
\mu_{0}\left\|u_{s}\right\|_{W^{1,2}(\Omega)}^{2} \leq & \left\|f_{0}\right\|_{L^{2}(\Omega)}\left\|u_{s}\right\|_{L^{2}(\Omega)}+\sum_{i=1}^{n}\left\|f_{i}\right\|_{L^{2}(\Omega)}\left\|\left(u_{s}\right)_{x_{i}}\right\|_{L^{2}(\Omega)} \\
& +\varepsilon c_{0}\left\|u_{s}\right\|_{W^{1,2}(\Omega)} \sum_{h=s}^{r}\left\|u_{h}\right\|_{W^{1,2}(\Omega)} .
\end{aligned}
$$

Thus, choosing $\varepsilon=\frac{\mu_{0}}{2 c_{0}}$ we have

$$
\left\|u_{s}\right\|_{W^{1,2}(\Omega)} \leq \frac{1}{\mu_{0}}\|f\|_{W^{-1,2}(\Omega)}+\frac{1}{2} \sum_{h=s}^{r}\left\|u_{h}\right\|_{W^{1,2}(\Omega)},
$$

for $s=1, \ldots, r$.

If we rewrite the last inequality for $s=r$ and we estimate $\left\|u_{r}\right\|_{W^{1,2}(\Omega)}$, then for $s=r-1$ and we estimate $\left\|u_{r-1}\right\|_{W^{1,2}(\Omega)}$ and so on, we get by substituting that

$$
\left\|u_{s}\right\|_{W^{1,2}(\Omega)} \leq \frac{2^{r-s+1}}{\mu_{0}}\|f\|_{W^{-1,2}(\Omega)},
$$

for $s=1, \ldots, r$.

Therefore, taking into account (2.6), we conclude that

$$
\|u\|_{W^{1,2}(\Omega)} \leq \sum_{s=1}^{r}\left\|u_{s}\right\|_{W^{1,2}(\Omega)} \leq\left(2^{r}-1\right) \frac{2}{\mu_{0}}\|f\|_{W^{-1,2}(\Omega)} .
$$

This, together with (2.12), ends the proof of the bound in (3.4).

Now, as it was already mentioned, it only remains to show that the operator

$$
L: u \in \stackrel{\circ}{W}^{1,2}(\Omega) \rightarrow L u \in W^{-1,2}(\Omega)
$$

is a Fredholm operator with zero index.

To this aim, set $\gamma=\sum_{i=1}^{n}\left(d_{i}-b_{i}\right)^{2}$ and denote by $\gamma u, u \in \stackrel{\circ}{W}^{1,2}(\Omega)$, the element of $W^{-1,2}(\Omega)$ given by

$$
\gamma u: v \in \stackrel{\circ}{W}^{1,2}(\Omega) \rightarrow \int_{\Omega} \gamma u v d x
$$

which is well defined in view of Lemma 2.1.

Then, consider the problem

$$
\left\{\begin{array}{l}
u \in \stackrel{\circ}{W}^{1,2}(\Omega), \\
L u+\frac{1}{2 v} \gamma u=f, \quad f \in W^{-1,2}(\Omega) .
\end{array}\right.
$$


Clearly, if we show that (3.6) has a unique solution, we end our proof, since in this case the operator $L$ can be seen as a sum between a Fredholm operator with zero index and a compact operator; and therefore, it is a Fredholm operator with zero index itself.

Indeed, we explicitly observe that the operator

$$
u \in \stackrel{\circ}{W}^{1,2}(\Omega) \rightarrow \gamma u \in W^{-1,2}(\Omega)
$$

is compact, since, by hypothesis $\left(h_{2}\right)$ and Lemma 2.1 , it is obtained as a composition between the compact operator

$$
u \in \stackrel{\circ}{W}^{1,2}(\Omega) \rightarrow \gamma^{1 / 2} u \in L^{2}(\Omega)
$$

and the bounded one

$$
v \in L^{2}(\Omega) \rightarrow \gamma^{1 / 2} v \in W^{-1,2}(\Omega),
$$

where $\gamma^{1 / 2} v, v \in L^{2}(\Omega)$, is the element of $W^{-1,2}(\Omega)$ defined by

$$
\gamma^{1 / 2} v: w \in \stackrel{\circ}{\mathscr{W}^{1,2}}(\Omega) \rightarrow \int_{\Omega} \gamma^{1 / 2} v w d x .
$$

To get the existence and uniqueness of the solution of problem (3.6), we want to make use of Lax-Milgram Lemma. Thus let us consider the bilinear form associated to it

$$
a(u, v)+\frac{1}{2 v} \int_{\Omega} \gamma u v d x, \quad u, v \in \stackrel{\circ}{W}^{1,2}(\Omega) .
$$

The continuity of the form (3.7) can be easily obtained by Lemma 2.1 . Considering the coercivity, for every $u \in \mathscr{W}^{1,2}(\Omega)$, in view of hypotheses $\left(h_{1}\right)$ and $\left(h_{3}\right)$, one has

$$
\begin{aligned}
a(u, u)= & \int_{\Omega} \sum_{i, j=1}^{n} a_{i j} u_{x_{i}} u_{x_{j}} d x+\int_{\Omega} \sum_{i=1}^{n}\left(b_{i}\left(u^{2}\right)_{x_{i}}+c u^{2}\right) d x \\
& +\int_{\Omega} \sum_{i=1}^{n}\left(d_{i}-b_{i}\right) u u_{x_{i}} d x \\
\geq & v\left\|u_{x}\right\|_{L^{2}(\Omega)}^{2}+\mu\|u\|_{L^{2}(\Omega)}^{2}+\int_{\Omega} \sum_{i=1}^{n}\left(d_{i}-b_{i}\right) u u_{x_{i}} d x .
\end{aligned}
$$

On the other hand, Hölder and Young inequalities give that

$$
\int_{\Omega} \sum_{i=1}^{n}\left|d_{i}-b_{i}\right|\left|u\left\|u_{x_{i}} \mid d x \leq \frac{v}{2}\right\| u_{x}\left\|_{L^{2}(\Omega)}^{2}+\frac{1}{2 v} \sum_{i=1}^{n}\right\|\left(d_{i}-b_{i}\right) u \|_{L^{2}(\Omega)}^{2}\right.
$$

and therefore,

$$
a(u, u)+\frac{1}{2 v} \int_{\Omega} \gamma u^{2} d x \geq \min \left\{\frac{v}{2}, \mu\right\}\|u\|_{W^{1,2}(\Omega)}^{2} .
$$

This concludes the proof of Theorem 3.1. 


\section{An a priori bound in $L^{p}$}

Here we want to prove, for a sufficiently regular $\operatorname{datum} f$, a $L^{p}$-a priori estimate, $p>2$, for a bounded solution of problem (3.1).

To this aim, we require a further assumption on the boundary of $\Omega$ :

$\Omega$ has the uniform $C^{1}$-regularity property.

Moreover, a technical lemma below is needed. We note that the proof of Lemma 4.1 follows the idea of the one of the estimate (3.4). However, in this case, there are some specific arguments that need to be explicitly treated.

Let $u_{s}$ be the functions of Lemma 2.2 corresponding to a fixed $u \in \stackrel{\circ}{W}^{1,2}(\Omega) \cap L^{\infty}(\Omega)$, to $g=\sum_{i=1}^{n}\left|d_{i}-b_{i}\right|$ and to a positive real number $\varepsilon$ to be specified in the proof of Lemma 4.1. The following result holds true:

Lemma 4.1 Let a be the bilinear form in (3.3). Under hypotheses $\left(h_{1}\right)-\left(h_{4}\right)$, there exists a constant $C \in \mathbb{R}_{+}$such that

$$
\left.\int_{\Omega}\left|u_{s}\right|^{p-2}\left(\left(u_{s}\right)_{x}^{2}+u_{s}^{2}\right) d x \leq C \sum_{h=s}^{r} a\left(u,\left|u_{h}\right|^{p-2} u_{h}\right), \quad s=1, \ldots, r, \forall p \in\right] 2,+\infty[
$$

where $C$ depends on $s, r, v, \mu$.

Proof Let $u, g, \varepsilon$ and $u_{s}$, for $s=1, \ldots, r$, be as above specified. Since $u \in L^{\infty}(\Omega)$, by definition of $u_{s}$ and by Lemma 2.2, the functions $u_{s} \in W^{1,2}(\Omega) \cap L^{\infty}(\Omega)$. Therefore, in view of hypothesis $\left(h_{4}\right)$, Lemma 3.2 in [17] applies giving that $\left|u_{s}\right|^{p-2} u_{s} \in \stackrel{\circ}{W^{1,2}}(\Omega)$ for any $p>2$.

Thus, we can take $\left|u_{s}\right|^{p-2} u_{s}$ as a test function in (3.3), obtaining by (2.9) that

$$
\begin{aligned}
a\left(u,\left|u_{s}\right|^{p-2} u_{s}\right)= & \int_{\Omega}\left[\sum_{i, j=1}^{n} a_{i j} u_{x_{i}}\left(\left|u_{s}\right|^{p-2} u_{s}\right)_{x_{j}}+\sum_{i=1}^{n} b_{i}\left(\left|u_{s}\right|^{p-2} u_{s} u\right)_{x_{i}}\right. \\
& \left.+c\left|u_{s}\right|^{p-2} u_{s} u+\sum_{i=1}^{n}\left(d_{i}-b_{i}\right) u\left(\left|u_{s}\right|^{p-2} u_{s}\right)_{x_{i}}\right] d x \\
= & \int_{\Omega}\left[(p-1)\left|u_{s}\right|^{p-2} \sum_{i, j=1}^{n} a_{i j}\left(u_{s}\right)_{x_{i}}\left(u_{s}\right)_{x_{j}}\right. \\
& +\sum_{i=1}^{n} b_{i}\left(\left|u_{s}\right|^{p-2} u_{s} u\right)_{x_{i}}+c\left|u_{s}\right|^{p-2} u_{s} u \\
& \left.+(p-1)\left|u_{s}\right|^{p-2} u \sum_{i=1}^{n}\left(d_{i}-b_{i}\right)\left(u_{s}\right)_{x_{i}}\right] d x .
\end{aligned}
$$

If we set

$$
\mu_{0}=\min \{v, \mu\}
$$

and

$$
H_{s}(u)=\left|u_{s}\right|^{p-2}\left(\left(u_{s}\right)_{x}^{2}+\left(u_{s}\right)^{2}\right),
$$


by hypotheses $\left(h_{1}\right)$ and $\left(h_{3}\right)$ and in view of $(2.7)$, one has

$$
\begin{aligned}
\mu_{0} \int_{\Omega} H_{s}(u) d x \leq & a\left(u,\left|u_{s}\right|^{p-2} u_{s}\right) \\
& +(p-1) \int_{\Omega} g\left|u_{s}\right|^{p-2}|u|\left(u_{s}\right)_{x} d x .
\end{aligned}
$$

On the other hand, by (2.6), (2.8) and (2.10), using the Hölder inequality, we get that there exists a constant $c_{0} \in \mathbb{R}_{+}$, such that

$$
\begin{aligned}
\int_{\Omega} g|u|\left|u_{s}\right|^{p-2}\left(u_{s}\right)_{x} d x & \leq \int_{\Omega} g|u||u|^{p / 2-1}\left|u_{s}\right|^{p / 2-1}\left(u_{s}\right)_{x} d x \\
& \leq c_{0} \sum_{h=s}^{r} \int_{\Omega} g\left|u_{h}\right|^{p / 2}\left|u_{s}\right|^{p / 2-1}\left(u_{s}\right)_{x} d x \\
& \leq c_{0}\left\|\left|u_{s}\right|^{p / 2-1}\left(u_{s}\right)_{x}\right\|_{L^{2}(\Omega)} \sum_{h=s}^{r}\left\|g\left|u_{h}\right|^{p / 2} \chi_{\operatorname{supp}\left(u_{s}\right)_{x}}\right\|_{L^{2}(\Omega)}
\end{aligned}
$$

with $c_{0}=c_{0}(r, p)$.

Thus, using hypothesis $\left(h_{2}\right)$, by Lemma 2.1 and (2.11), we obtain

$$
\begin{aligned}
& \int_{\Omega} g|u|\left|u_{s}\right|^{p-2}\left(u_{s}\right)_{x} d x \\
& \quad \leq c_{1}\left\|\left|u_{s}\right|^{p / 2-1}\left(u_{s}\right)_{x}\right\|_{L^{2}(\Omega)}\left\|g \chi_{\operatorname{supp}\left(u_{s}\right)_{x}}\right\|_{M^{2 t, \lambda}(\Omega)} \sum_{h=s}^{r}\left\|\left|u_{h}\right|^{p / 2}\right\|_{W^{1,2}(\Omega)} \\
& \quad \leq c_{1} \varepsilon\left\|\left|u_{s}\right|^{p / 2-1}\left(u_{s}\right)_{x}\right\|_{L^{2}(\Omega)} \sum_{h=s}^{r}\left\|\left|u_{h}\right|^{p / 2}\right\|_{W^{1,2}(\Omega)}
\end{aligned}
$$

with $c_{1}=c_{1}(r, p, n, t)$.

Now, we observe that explicit calculations give

$$
\left\|\left|u_{h}\right|^{p / 2}\right\|_{W^{1,2}(\Omega)} \leq \frac{p}{2}\left(\int_{\Omega} H_{h}(u) d x\right)^{1 / 2}, \quad h=s, \ldots, r .
$$

Hence, putting together (4.3), (4.4) and (4.5), we get

$$
\begin{aligned}
\int_{\Omega} H_{s}(u) d x \leq & \frac{1}{\mu_{0}} a\left(u,\left|u_{s}\right|^{p-2} u_{s}\right) \\
& +\frac{c_{2}}{\mu_{0}} \varepsilon\left(\int_{\Omega} H_{s}(u) d x\right)^{1 / 2} \sum_{h=s}^{r}\left(\int_{\Omega} H_{h}(u) d x\right)^{1 / 2},
\end{aligned}
$$

with $c_{2}=c_{2}(r, p, n, t)$.

Thus, by Young inequality,

$$
\begin{aligned}
\int_{\Omega} H_{s}(u) d x & \leq \frac{1}{\mu_{0}} a\left(u,\left|u_{s}\right|^{p-2} u_{s}\right)+\frac{c_{3}}{\mu_{0}} \varepsilon\left(\int_{\Omega} H_{s}(u) d x\right)^{1 / 2}\left(\sum_{h=s}^{r} \int_{\Omega} H_{h}(u) d x\right)^{1 / 2} \\
& \leq \frac{1}{\mu_{0}} a\left(u,\left|u_{s}\right|^{p-2} u_{s}\right)+\frac{c_{3}}{\mu_{0}}\left(\frac{\eta}{2} \int_{\Omega} H_{s}(u) d x+\frac{\varepsilon^{2}}{2 \eta} \sum_{h=s}^{r} \int_{\Omega} H_{h}(u) d x\right),
\end{aligned}
$$


with $c_{3}=c_{3}(r, p, n, t)$.

Choosing $\eta=\frac{\mu_{0}}{c_{3}}$ and $\varepsilon=\frac{\mu_{0}}{c_{3} \sqrt{2}}$ we have

$$
\int_{\Omega} H_{s}(u) d x \leq \frac{2}{\mu_{0}} a\left(u,\left|u_{s}\right|^{p-2} u_{s}\right)+\frac{1}{2} \sum_{h=s}^{r} \int_{\Omega} H_{h}(u) d x
$$

$s=1, \ldots, r$.

If we rewrite the last inequality for $s=r$, then for $s=r-1$ and take into account the estimate of $\int_{\Omega} H_{r}(u) d x$ obtained in the previous step, and so on, we conclude our proof. Indeed, we get

$$
\int_{\Omega} H_{s}(u) d x \leq C \sum_{h=s}^{r} a\left(u,\left|u_{h}\right|^{p-2} u_{h}\right)
$$

with $C=C\left(s, r, \mu_{0}\right)$.

We are finally in position to prove the above mentioned $L^{p}$-bound.

Theorem 4.2 Assume that the hypotheses $\left(h_{1}\right)-\left(h_{4}\right)$ are satisfied. Iff is in $L^{2}(\Omega) \cap L^{\infty}(\Omega)$ and the solution $u$ of $(3.1)$ is in $\stackrel{\circ}{W^{1,2}}(\Omega) \cap L^{\infty}(\Omega)$, then

$$
\left.\|u\|_{L^{p}(\Omega)} \leq C\|f\|_{L^{p}(\Omega)} \quad \forall p \in\right] 2,+\infty[
$$

where $C$ is a constant depending on $n, t, p, v, \mu,\left\|d_{i}-b_{i}\right\|_{M^{2 t, \lambda}(\Omega)}, i=1, \ldots, n$.

Proof Fix $p \in] 2,+\infty$ [. If we consider the functions $u_{s}, s=1, \ldots, r$, corresponding to the solution $u$, to $g$ and $\varepsilon$ as in Lemma 4.1, easy computations together with (2.6) give that

$$
\int_{\Omega}|u|^{p} d x \leq c_{0} \sum_{s=1}^{r} \int_{\Omega}\left|u_{s}\right|^{p} d x
$$

with $c_{0}=c_{0}(r, p)$.

Thus, by (4.1), one has

$$
\int_{\Omega}|u|^{p} d x \leq c_{0} \sum_{s=1}^{r} C_{s} \sum_{h=s}^{r} a\left(u,\left|u_{h}\right|^{p-2} u_{h}\right) \leq c_{1} \sum_{s=1}^{r} a\left(u,\left|u_{s}\right|^{p-2} u_{s}\right),
$$

with $C_{s}=C_{s}(s, r, \nu, \mu)$ and $c_{1}=c_{1}(r, p, \nu, \mu)$.

Hence by (2.8) and Hölder inequality, we get

$$
\begin{aligned}
\|u\|_{L^{p}(\Omega)}^{p} & \leq c_{1} \sum_{s=1}^{r} \int_{\Omega} f\left|u_{s}\right|^{p-2} u_{s} d x \\
& \leq r c_{1} \int_{\Omega}\left|f\left\|\left.u\right|^{p-1} d x \leq r c_{1}\right\| f\left\|_{L^{p}(\Omega)}\right\| u \|_{L^{p}(\Omega)}^{p-1} .\right.
\end{aligned}
$$

This concludes the proof, in view of (2.12). 


\section{Competing interests}

The authors declare that they have no competing interests.

\section{Author's contributions}

The authors conceived and wrote this article in collaboration and with the same responsibility. Both of them read and approved the final manuscript.

\section{Acknowledgement}

The authors would like to thank anonymous referees for a careful reading of this article and for valuable suggestions and comments.

Received: 27 February 2012 Accepted: 15 June 2012 Published: 28 June 2012

\section{References}

1. Chicco, M: An a priori inequality concerning elliptic second order partial differential equations of variational type. Matematiche 26, 173-182 (1971)

2. Gilbarg, D, Trudinger, NS: Elliptic Partial Differential Equations of Second Order, 2nd edn. Springer, Berlin (1983)

3. Ladyzhenskaja, OA, Ural'tzeva, NN: Equations aux Derivèes Partielles de Type Elliptique. Dunod, Paris (1966)

4. Miranda, C: Alcune osservazioni sulla maggiorazione in $L^{v}$ delle soluzioni deboli delle equazioni ellittiche del secondo ordine. Ann. Mat. Pura Appl. 61, 151-169 (1963)

5. Stampacchia, G: Le problème de Dirichlet pour les équations elliptiques du second ordre à coefficients discontinus. Ann. Inst. Fourier (Grenoble) 15, 151-169 (1966)

6. Trudinger, NS: Linear elliptic operators with measurable coefficients. Ann. Sc. Norm. Super. Pisa, Cl. Sci. 27, 265-308 (1973)

7. Bottaro, G, Marina, ME: Problema di Dirichlet per equazioni ellittiche di tipo variazionale su insiemi non limitati. Boll. Unione Mat. Ital. 8, 46-56 (1973)

8. Transirico, M, Troisi, M: Equazioni ellittiche del secondo ordine a coefficienti discontinui e di tipo variazionale in aperti non limitati. Boll. Unione Mat. Ital, B 2, 385-398 (1988)

9. Transirico, M, Troisi, M, Vitolo, A: Spaces of Morrey type and elliptic equations in divergence form on unbounded domains. Boll. Unione Mat. Ital, B 9, 153-174 (1995)

10. Monsurrò, S, Transirico, M: A $L^{p}$-estimate for weak solutions of elliptic equations. Abstr. Appl. Anal. (2012). doi:10.1155/2012/376179

11. Chicco, M, Venturino, M: Dirichlet problem for a divergence form elliptic equation with unbounded coefficients in an unbounded domain. Ann. Mat. Pura Appl. 178, 325-338 (2000)

12. Lions, PL: Remarques sur les équations linéaires elliptiques du second ordre sous forme divergence dans les domaines non bornés. Atti Accad. Naz. Lincei, Rend. Cl. Sci. Fis. Mat. Nat. 78, 205-212 (1985)

13. Lions, PL: Remarques sur les équations linéaires elliptiques du second ordre sous forme divergence dans les domaines non bornés II. Atti Accad. Naz. Lincei, Rend. Cl. Sci. Fis. Mat. Nat. 79, 178-183 (1985)

14. Caso, L, D'Ambrosio, R, Monsurrò, S: Some remarks on spaces of Morrey type. Abstr. Appl. Anal. (2010) doi:10.1155/2010/242079

15. Cavaliere, P, Longobardi, M, Vitolo, A: Imbedding estimates and elliptic equations with discontinuous coefficients in unbounded domains. Matematiche 51, 87-104 (1996)

16. Stampacchia, G: Equations elliptiques du second ordre à coefficients discontinus. Séminaire de mathématiques supérieures, Université de Montréal, $4^{e}$ session, été 1965. Les presses de l'Université de Montréal, Montreal (1966)

17. Caso, L, Cavaliere, $P$, Transirico, M: Solvability of the Dirichlet problem in $W^{2, p}$ for elliptic equations with discontinuous coefficients in unbounded domains. Matematiche 57, 287-302 (2002)

doi:10.1186/1687-2770-2012-67

Cite this article as: Monsurrò and Transirico: Dirichlet problem for divergence form elliptic equations with discontinuous coefficients. Boundary Value Problems 2012 2012:67.

\section{Submit your manuscript to a SpringerOpen ${ }^{\circ}$ journal and benefit from:}

- Convenient online submission

- Rigorous peer review

- Immediate publication on acceptance

- Open access: articles freely available online

- High visibility within the field

- Retaining the copyright to your article 